\title{
Multi-Wavelength Studies of the GX 339-4 2010 outburst
}

\author{
Marion Cadolle Bel ${ }^{1}$, S. Corbel ${ }^{2}$, A. Veledina ${ }^{3}$, J. Rodriguez ${ }^{2}$, \\ P. D’Avanzo ${ }^{4}$, J. Tomsick ${ }^{5}$, D. M. Russell ${ }^{6}$ and F. Lewis ${ }^{7}$ \\ ${ }^{1}$ ESAC/ISOC, Spain; email: Marion.Cadolles@sciops.esa.int; ${ }^{2}$ AIM \& CEA-Saclay, \\ France; ${ }^{3}$ Oulu Univ., Finland; ${ }^{4}$ INAF, Italy; ${ }^{5}$ SSL/California Univ., USA; ${ }^{6}$ Tenerife Obs., \\ Spain; ${ }^{7}$ FT project, Glamorgan Univ., Wales
}

\begin{abstract}
The microquasar GX 339-4 experienced an outburst in 2010. We focus on observations that are quasi-simultaneous with those made by INTEGRAL and RXTE in March-April 2010 with radio, NIR, optical and UV data. X-ray transients are extreme systems, often harboring a black hole, known to emit throughout the whole electromagnetic spectrum in outburst. We studied the source evolution and correlated changes in all wavelengths. The bolometric flux increased from 0.8 to $2.9 \times 10^{-8} \mathrm{erg} \mathrm{cm}^{-2} \mathrm{~s}^{-1}$ while the relative contribution of the hot medium decreased. The radio, NIR and optical emission from jets was detected and observed to fade as the source softened; reprocessing in the disc was strong at the end.
\end{abstract}

Keywords. Black hole physics, stars: GX 339-4 gamma rays, IR, radio: general, X-rays: binaries

\section{Introduction}

X-ray transients (XTs) are accreting low-mass X-ray binaries (LMXBs) that spend most of their time in a faint, quiescent state. They undergo large amplitude outbursts with rise times of only a few days or weeks (or months in the case of GX 339-4), with typical recurrence periods of many years (Tanaka \& Shibazaki 1996). Based on the relative strengths of each spectral component, the degree of variability, shape of the PDS and on the properties of the radio emission in outburst, different spectral states have been defined (e.g., the low/hard (LHS) and the high/soft (HSS) main canonical states, McClintock \& Remillard 2006; Homan \& Belloni 2005). GX 339-4 is a recurrent XT with regular outbursts. We triggered INTEGRAL during the initial hard X-ray phase (Tomsick 2010; Prat et al. 2010), and in the declining phase (Cadolle Bel et al. 2010). We report and discuss some of the results of this multiwavelength campaign.

\section{Observations and Data Analysis}

Figure 1 (left) shows the RXTE/ASM, SWIFT/BAT X-ray and INTEGRAL light curves of the outburst (other multi-wavelength observations indicated). The IBIS/ISGRI and JEM-X data were reduced using standard analysis procedures of the off-line scientific analysis OSA 9.0 following standard procedures (e.g., Rodriguez et al. 2008; Cadolle Bel et al. 2009). The RXTE data were reduced with the HEASOFT software package v6.10, following standard procedures (see Rodriguez et al. 2008). All Swift/XRT observations discussed in this paper were made in window timing (WT) mode to avoid pile-up due to the source brightness. In addition, data were collected from the Swift/UVOT (UV and optical) instrument, with MJD spanning from 55217 to 55310 . Optical and NIR observations were performed with the REM telescope (see, e.g., Zerbi \& The Rem Team 2001; Chincarini et al. 2003; Covino et al. 2004); optical data were also collected using the 

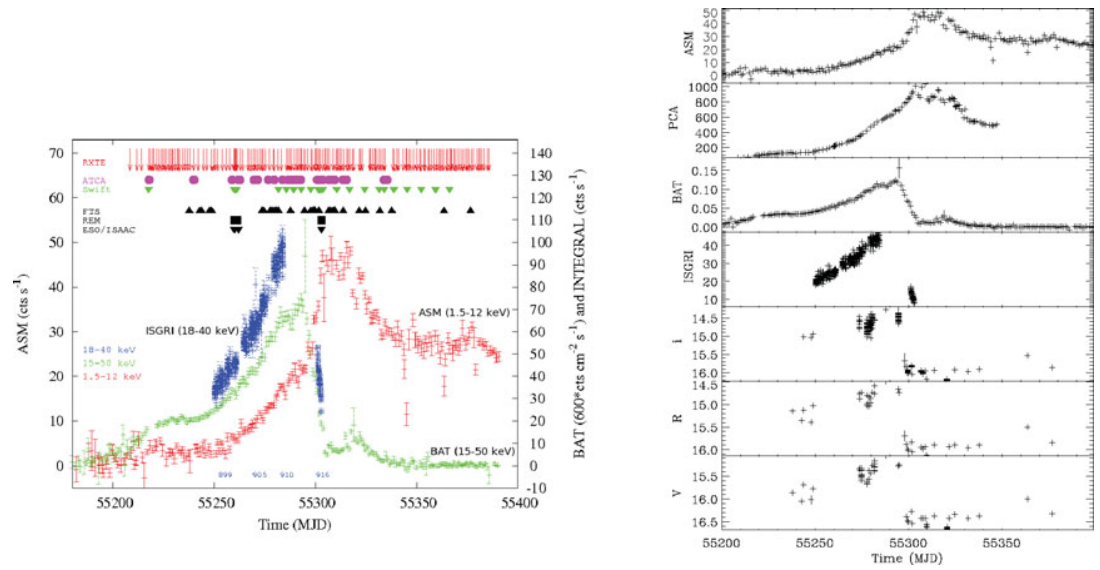

Figure 1. Left: Daily RXTE/ASM, Swift/BAT and INTEGRAL/ISGRI light curves of GX 339-4 during the 2010 outburst. Other observations performed with ATCA, the FTS, the REM/ROSS, REMIR and the ESO/ISAAC telescopes are indicated.The numbers near the bottom of the figure are INTEGRAL revolutions. Right: RXTE/ASM (cts s ${ }^{-1}$ in $1.5-12 \mathrm{keV}$ ), PCA (cts s${ }^{-1}$ in 3-30 keV), Swift/BAT $\left(\right.$ cts cm $^{-2} \mathrm{~s}^{-1}$ in 15-50 keV), INTEGRAL/ISGRI (cts $\mathrm{s}^{-1}$ in $40-80 \mathrm{keV}$ ) and FTS light curves (filters $V, R$ and $i^{\prime}$ ).
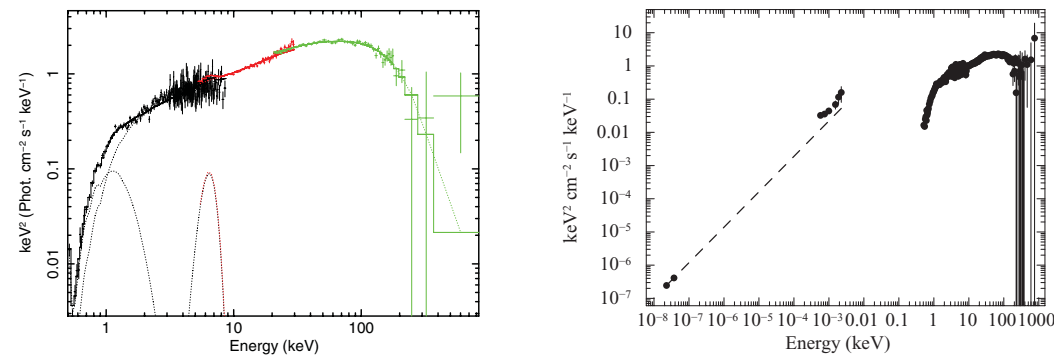

Figure 2. Left: Swift/XRT, RXTE/PCA and INTEGRAL/ISGRI LHS spectra of GX 339-4 (MJD 55259.9-55261.1; see text for fits). Right: SED from radio to soft $\gamma$-ray data, MJD $\sim 55259.9-55261.1$ (Rev. 902). The dashed line is a simple power-law extrapolation of the two radio data points up to the NIR frequencies (this is not a physical model, but only shown for visual purpose).

2-metre robotic Asutralian Faulkes Telescope South (FTS). All science images were produced using an automatic pipeline that de-biases and flat-fields the raw images. Finally, GX 339-4 was frequently observed at radio frequencies with the ATCA; fluxes were $9.02 \pm 0.10 \mathrm{mJy}, 9.56 \pm 0.05 \mathrm{mJy}$ on MJD 55261.89 then $8.16 \pm 0.05 \mathrm{mJy}$ and $7.94 \pm 0.10$ mJy on MJD 55262.91 at, respectively, 5.5 and $9.0 \mathrm{GHz}$.

\section{Results}

Fig. 1 (right) presents the light curves obtained with the ASM (2-10 keV), PCA (3$30 \mathrm{keV}$ ), BAT (15-40 keV), ISGRI (40-80 keV) and FTS ( $V, R$ and $i^{\prime}$ magnitudes) instruments. The source shows the typical behaviour of an XT: the outburst started in hard X-rays probably around MJD 55199. Around MJD 55295, the hard X-rays reached their peak and the emission began to decrease (with a secondary peak at MJD 55319 ) while the source continued to increase in soft X-rays until MJD 55327, before decreasing. This behaviour indicates a state transition from a hard to a softer state. The optical also dropped at the start of the transition, as reported in previous outbursts (Homan et al. 2005; Coriat et al. 2009). While during MJD 55259.9-55261.1 (Rev. 902) the source was still in a hard state, during MJD 55301.1-55303.3 (Rev. 916) GX 339-4 
Table 1. Best-fit spectral parameters around the period of our main INTEGRAL observations.

\begin{tabular}{|c|c|c|c|c|c|c|c|c|c|c|}
\hline $\begin{array}{c}\text { Time } \\
(\mathrm{M} \mathrm{JD}-55000)\end{array}$ & $\begin{array}{c}\text { Observations } \\
\text { (Rev. \#) }\end{array}$ & Disc Norm. & $\begin{array}{l}k T_{\text {in }} \\
(\mathrm{keV})\end{array}$ & $\begin{array}{c}k T_{\mathrm{e}}(\mathrm{keV}) \\
\quad \text { or } \Gamma\end{array}$ & $\tau$ & $\begin{array}{c}E_{\mathrm{Fe}} \text { line } \\
(\mathrm{keV})\end{array}$ & $\omega / 2 \pi$ & $\begin{array}{l}\chi_{\text {red }}^{2} \\
(\mathrm{~d} \text { of })\end{array}$ & $F^{a}$ & $F_{\text {bol }}^{b}$ \\
\hline $217.7-217.8$ & N.A. & $\begin{array}{c}33784 \\
17644\end{array}$ & $0.18 \pm 0.01$ & $\Gamma=1.62 \pm 0.02$ & - & $6.53_{-0.35}^{+0.32}$ & $0.34_{-0.08}^{+0.10}$ & $1.45(281)$ & 1.43 & N.A. \\
\hline $237.5-240.2$ & $895^{c}$ & $\begin{array}{l}35+20 \\
-9\end{array}$ & $0.83 \pm 0.10$ & $41_{-4}^{+5}$ & $1.63_{-0.17}^{+0.14}$ & $6.56_{-0.25}^{+0.20}$ & $0.25 \pm 0.05$ & $0.84(97)$ & 1.99 & 0.82 \\
\hline $240.7-243.2$ & $896^{c}$ & $\begin{array}{l}100_{-50}^{+9}+186 \\
-{ }_{-50}\end{array}$ & $0.67_{-0.11}^{+0.02}$ & $38_{-3}^{+4}$ & $1.69_{-0.14}^{+0.13}$ & $\begin{array}{r}+0.25 \\
6.15_{-0.15}^{+0.21}\end{array}$ & $0.19_{-0.04}^{+0.05}$ & $1.05(102)$ & 2.19 & 0.89 \\
\hline $243.7-246.2$ & $897^{c}$ & $100 \pm 50$ & $0.65 \pm 0.10$ & $41_{-3}^{+5}$ & $1.53_{-0.15}^{+0.12}$ & $6.00+0.14$ & $0.21 \pm 0.03$ & $1.13(103)$ & 2.14 & 0.86 \\
\hline $246.7-249.1$ & $898^{c}$ & $100_{-50}^{+91}$ & $0.64_{-0.07}^{+0.03}$ & $39_{-3}^{+6}$ & $1.59_{-0.15}^{+0.14}$ & $6.00+0.25$ & $0.22 \pm 0.05$ & $1.14(102)$ & 2.29 & 0.92 \\
\hline $249.7-252.1$ & $899^{c}$ & $\begin{array}{l}66^{+252} \\
-29\end{array}$ & $0.75+0.14$ & $40 \begin{array}{l}+5 \\
-3\end{array}$ & $1.59+0.13$ & $6.37 \pm 0.20$ & $0.26 \pm 0.05$ & $1.09(102)$ & 2.49 & 1.02 \\
\hline $252.5-255.1$ & $900^{c}$ & $102 \pm 51$ & $0.68_{-0.19}^{+0.13}$ & $\begin{array}{l}39+4 \\
-3\end{array}$ & $1.59_{-0.15}^{+0.13}$ & $6.17_{-0.49}^{+0.23}$ & $0.25_{-0.04}^{+0.05}$ & $0.78(102)$ & 2.74 & 1.09 \\
\hline $255.6-258.1$ & $901^{c}$ & $124_{-56}^{+388}$ & $0.70_{-0.16}^{+0.11}$ & $45_{-4}^{+7}$ & $1.36_{-0.17}^{+0.14}$ & $6.31_{-0.70}^{+0.30}$ & $0.32 \pm 0.04$ & $0.99(102)$ & 3.18 & 1.25 \\
\hline $259.9-261.1$ & 902 & $82887_{-38606}^{+63704}$ & $0.19 \pm 0.01$ & $\begin{array}{l}38_{-2}^{+3} \\
-2\end{array}$ & $1.49^{+0.10}-0.11$ & $\begin{array}{r}6.23+0.15 \\
-0.23\end{array}$ & $0.25 \pm 0.04$ & $1.58(333)$ & 3.38 & 1.64 \\
\hline $264.5-267.1$ & $904^{c}$ & $134_{-57}^{+441}$ & $\begin{array}{l}0.73_{-0.18}^{+0.12} \\
-0.18\end{array}$ & $\begin{array}{l}39^{+2} \\
-3\end{array}$ & $1.45_{-0.13}^{+0.11}$ & $\begin{array}{r}-0.23 \\
6.23+0.29 \\
-0.66\end{array}$ & $0.34_{-0.04}^{+0.05}$ & $1.07(102)$ & 4.21 & 1.51 \\
\hline $267.6-270.1$ & $905^{c}$ & $183_{-87}^{+1406}$ & $0.67_{-0.20}^{+0.12}$ & $41_{-3}^{+5}$ & $1.31_{-0.14}^{+0.12}$ & $6.27_{-0.70}^{+0.27}$ & $0.35 \pm 0.04$ & $1.11(102)$ & 4.60 & 1.61 \\
\hline $270.4-272.8$ & $906^{c}$ & $\begin{array}{l}370+1664 \\
-199\end{array}$ & $0.60_{-0.13}^{+0.11}$ & $\begin{array}{l}38+4 \\
38\end{array}$ & $1.40_{-0.12}^{+0.11}$ & $6.07_{-0.47}^{+0.30}$ & $0.32_{-0.03}^{+0.04}$ & $1.33(102)$ & 5.04 & 1.77 \\
\hline $273.4-276.0$ & $907^{c}$ & $\begin{array}{c}198+568 \\
-71\end{array}$ & $0.68_{-0.15}^{+0.09}$ & $\begin{array}{l}39+4 \\
-3\end{array}$ & $1.28_{-0.13}^{+0.11}$ & $6.45_{-0.50}^{+0.19}$ & $0.35+0.05$ & $1.59(102)$ & 5.67 & 1.98 \\
\hline $276.4-278.8$ & $908^{c}$ & $338_{-174}^{+2463}$ & $0.62+0.13$ & $37_{-3}^{+4}$ & $1.28+0.11$ & $6.29_{-0.65}^{+0.35}$ & $0.39 \pm 0.06$ & $1.15(102)$ & 6.59 & 2.15 \\
\hline $279.4-281.5$ & 909 & $\begin{array}{r}32816_{-19118}^{+33761} \\
-29\end{array}$ & $0.21_{-0.01}^{+0.02}$ & $\begin{array}{l}39+4 \\
+3\end{array}$ & $1.16_{-0.13}^{+0.11}$ & $6.23_{-0.25}^{+0.15}$ & $0.39 \pm 0.04$ & $0.88(312)$ & 7.80 & 2.79 \\
\hline $281.7-281.8$ & N.A. & $\begin{array}{l}52+24 \\
-20\end{array}$ & $0.85 \pm 0.09$ & $\Gamma=1.80 \pm 0.01$ & - & $6.27_{-0.22}^{+0.16}$ & $0.38 \pm 0.06$ & $0.57(255)$ & 7.94 & N.A. \\
\hline $282.4-284.9$ & $910^{c}$ & $32_{-6}^{+23}$ & $1.19_{-0.11}^{+0.10}$ & $40_{-3}^{+4}$ & $1.14 \pm 0.12$ & $6.00+0.58$ & $0.49 \pm 0.04$ & $0.96(96)$ & 7.69 & 2.25 \\
\hline $289.3-289.6$ & N.A. & $840436+118692$ & $0.16_{-0.01}^{+0.02}$ & $\Gamma=1.79 \pm 0.03$ & - & $6.14_{-0.29}^{+0.21}$ & $0.28 \pm 0.07$ & $1.34(228)$ & 9.65 & N.A. \\
\hline $297.8-297.9$ & N.A. & $174096_{-62284}^{+86620}$ & $0.22 \pm 0.01$ & $\Gamma=1.95 \pm 0.02$ & - & $6.00+0.04$ & $0.10 \pm 0.05$ & $1.32(260)$ & 11.4 & N.A. \\
\hline $301.1-303.3$ & 916 & $\begin{array}{l}3196+367 \\
-288\end{array}$ & $0.61 \pm 0.02$ & $256{ }_{-83}^{+6}$ & $0.01_{-0.01}^{+0.02}$ & $6.00+0.22$ & $0.51_{-0.10}^{+0.09}$ & $1.91(252)$ & 12.2 & 2.93 \\
\hline $303.6-303.8$ & N.A. & $2652+526$ & $0.73 \pm 0.04$ & $\begin{array}{l}35 \\
-5 \\
-14\end{array}$ & $0.50+0.28$ & $6.74_{-0.14}^{+0.16}$ & $0.36_{-0.32}^{+0.44}$ & $1.77(192)$ & 14.6 & N.A. \\
\hline $310.6-310.7$ & N.A. & $\begin{array}{l}999_{-44}^{+45} \\
\end{array}$ & $1.00_{-0.01}^{+0.02}$ & $\Gamma=2.33_{-0.07}^{+0.06}$ & - & $6.15_{-0.09}^{+0.08}$ & - & $1.50(178)$ & 13.3 & N.A. \\
\hline
\end{tabular}

Notes: Models applied in XSPEC notations: CONSTANT* PHABS*REFLECT*(DISKBB+GAUSSIAN+POWERLAW) or CONSTANT*PHABS*REFLECT*(DISKBB+GAUSSIAN+COMPTT) (not all components always needed). $\Gamma$ is the photon index. $N_{\mathrm{H}}$ varied between $4.9-6.3 \times 10^{21} \mathrm{~cm}^{-2}$. Errors are given at the $90 \%$ confidence level $\left(\Delta \chi^{2}=2.7\right)$. a) Computed in the $2-20 \mathrm{keV}$ range. Units: $10^{-9} \mathrm{erg} \mathrm{cm}^{-2} \mathrm{~s}^{-1}$. b) X-ray bands extrapolated in the $0.01 \mathrm{keV}-10 \mathrm{MeV}$ range. Units: $10^{-8} \mathrm{erg} \mathrm{cm}^{-2} \mathrm{~s}^{-1}$. c) XRT data not available. See Cadolle Bel et al. (2011) for more details.

was extremely bright in Swift/XRT (> 400 cts s $^{-1}$ ). The JEM-X fluxes were around 370 mCrab and $240 \mathrm{mCrab}$ in, respectively, the 3-10 and 10-20 keV energy bands, while the ISGRI fluxes were varying around $190 \mathrm{mCrab}$ and $130 \mathrm{mCrab}$ in the $20-40 \mathrm{keV}$ and 40-80 keV energy bands. The X-ray source was softening. We fitted the closest XRT, PCA and IBIS/ISGRI spectra simultaneously with XSPEC v12.6.0 (Arnaud 1996). The data were in general well-fitted using absorbed power-law (or cut-off power-law and Comptonisation) with reflection combined with a multicolour black-body, and a Gaussian Fe line at $\sim 6.5 \mathrm{keV}$. An example of the fitted spectra obtained during our INTEGRAL observations is shown in Fig. 2 (left). Table 1 summarizes the main spectral parameters for all observations. GX 339-4 reached the brightest magnitudes recorded on 2010 April 1, MJD 55287, just before the start of the transition from the LHS to softer states. During this LHS rise, the source brightened at a mean rate of $\sim 0.01 \mathrm{mag} \mathrm{d}^{-1}$

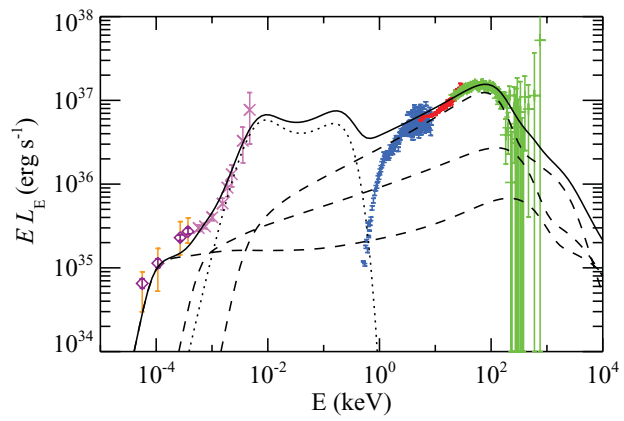

Figure 3. SED with the same data than in Figure 2 (right) fitted with the model of Veledina et al. (2012). Work in progress. between MJD 55237-55287. Between MJD 55294-55297, the optical flux faded rapidly by $\sim 1$ mag in 3.0 days (Russell et al. 2010) and a change in the SED to a bluer colour was observed. Months later, the optical and NIR brightnesses in all filters decreased. ATCA observations conducted on MJD 55283 showed flux densities $\sim 20$ mJy with an inverted radio spectrum (spectral index spanning 
+0.1 to +0.2 , typical of powerful self-absorbed compact jets observed in LHS). On MJD 55372 , no radio emission was detected at the location of GX 339-4: this was consistent with the source being in a soft state (Corbel et al. 2010). We refer to Cadolle Bel et al. (2011) for more details on the fitting and spectral parameter evolutions.

\section{Discussion}

Spectral analysis showed that the source transited from an initial LHS, with a spectrum dominated by Comptonisation in the beginning of the outburst (MJD 55217- 55281), to softer states where the power-law cut-off was not needed anymore and the photon index of the power-law was very soft $(\sim 2.3)$. In X-ray binaries, a cut-off power-law spectrum is usually interpreted as the signature of inverse Comptonisation of soft-seed photons by a thermalised population of electrons. In the LHS, we detected the compact core jet also in the optical/IR. We added radio flux data to the SED in Fig. 2 (right): it is remarkably similar to the Fig. 2 of Corbel \& Fender (2002). One can interpret the results with a very rough approach: a simple power-law extrapolation of the radio data up to the NIR/optical clearly does not fit the data since an excess emission is seen. Similarly, several power-law components with distinct slopes are needed to fit later SEDs: after the LHS, the radio spectral index became typical of optically thin synchrotron radiation, probably as a result of freely expanding plasma blobs previously ejected (see, e.g., Fender et al. 2004); multiple ejection events took place during the outburst and then interacted with the interstellar medium (Corbel et al. 2010). Besides, we applied a hot flow model (Fig. 3) to describe the broadband spectra around MJD 55260. Finally, we clearly detected the jet evolution and its dramatic quenching at all wavelengths (for more details: Cadolle Bel et al. 2011, 2012).

\section{References}

Arnaud, K. A. 1996, ASP Conferences, 101, 17

Cadolle Bel, M., Kuulkers, E., Ibarra, A., et al. 2010, The Astronomer's Telegram, 2573, 1

Cadolle Bel, M., Prat, L., Rodriguez, J., et al. 2009, A\& A, 501, 1

Cadolle Bel, M., Rodriguez, J., D'Avanzo, P., et al. 2011, A\&SA, 534, A119

Cadolle Bel, M., Rodriguez, J., D’Avanzo, P., et al. 2012, A\&SA, 544, C2

Chincarini, G., Zerbi, F., Antonelli, A., et al. 2003, The Messenger, 113, 40

Corbel, S., Broderick, J., Calvelo, D., et al. 2010, The Astronomer's Telegram, 2745, 1

Corbel, S. \& Fender, R. P. 2002, ApJ, 573, L35

Coriat, M., Corbel, S., Buxton, M. M., et al. 2009, MNRAS, 400, 123

Covino, S., Stefanon, M., Sciuto, G., et al. 2004, in Presented at the Society of Photo-Optical Instrumentation Engineers (SPIE) Conference, Vol. 5492, Society of Photo-Optical Instrumentation Engineers (SPIE) Conference Series, ed. A. F. M. Moorwood \& M. Iye, 1613-1622

Fender, R. P., Belloni, T. M., \& Gallo, E. 2004, MNRAS, 355, 1105

Homan, J. \& Belloni, T. 2005, Astrophysics and Space Science, 300, 107

Homan, J., Buxton, M., Markoff, S., et al. 2005, ApJ, 624, 295

McClintock, J. E. \& Remillard, R. A. 2006, Black hole binaries, Compact stellar X-ray sources. Edited by Walter Lewin \& Michiel van der Klis: Cambridge University Press, 157

Prat, L., Cadolle Bel, M., Terrier, R., et al. 2010, The Astronomer's Telegram, 2455, 1

Rodriguez, J., Shaw, S. E., Hannikainen, D. C., et al. 2008, ApJ, 675, 1449

Russell, D. M., Buxton, M., Lewis, F., \& Altamirano, D. 2010, The Astronomer's Telegram, 2547,1

Tanaka, Y. \& Shibazaki, N. 1996, ARAA, 34, 607

Tomsick, J. A. 2010, The Astronomer's Telegram, 2384, 1

Veledina, A., Poutanen, J., \& Vurm, I. 2012, ArXiv e-prints

Zerbi, F. M. \& The Rem Team. 2001, in Astronomische Gesellschaft Meeting Abstracts, Vol. 18, Astronomische Gesellschaft Meeting Abstracts, ed. E. R. Schielicke, J101+ 